\title{
Applications of Multimedia in Solfeggio Teaching
}

\author{
Bin $\mathrm{Li}$ \\ School of Music \\ University of Jinan \\ Jinan, China 250022
}

\begin{abstract}
Traditionally the solfeggio teaching is single, yet modern multimedia technique audio hardware support and VSTi and DXi software may expand traditional teaching means. Based on the interaction, integration and real time of multimedia technique, this article discusses the solfeggio training mode, showing that multimedia make it possible for multi-timbre training, besides, it can help keep exams and assessments quantitative, standard and impersonal, the multimedia teaching is an efficient tool to improve students' interests in training, changing traditional mode in the solfeggio teaching as well as improving training modes and contents.
\end{abstract}

Keywords-multimedia technique; teaching; MIDI; VSTi

\section{INTRODUCTION}

What to learn in solfeggio is an important part of basic music skills training, a necessary capability that music workers should have and a required course in traditional basic music teaching. With the rapid development of modern educational technique and means, the application of advanced teaching hardware and software can greatly improve teaching methods and expand training contents, making the goal of traditional solfeggio better meet current professional environment.

Traditional solfeggio training is aimed at training the capabilities of professional students in listening and playing in order to improve their understanding and manifestation in music. The most important points to feel sounds are pitch, intensity, volume and length. Of which, the pitch, intensity and length are main contents in traditional training, which can be exercised independently, though the intensity is easy to distinguish in feeling sound, the training of relative volume of music sound (melodic accent) is done through other three trainings. Specific training contents mainly include melodic repetition, single-tone listening, chord listening, rhythm listening, melody listening and so on, piano is a main training tool in traditional teaching. With regard to traditional music training methods, piano has more advantages than other musical instruments such as range of musical instruments namely the whole musical-tone system of music, playing directly and visually. However, the limitations of piano also restrict the teaching such as single ton, no continuous long tone, as well as teachers' playing technique and more. During training, the long term dependence on piano has caused a fixed basic mode in teaching, and the single teaching has seriously influenced students' acceptance speed and understanding in professional training.
Computer-aided multimedia technique refers to an IT to achieve interactive processing of multimedia through computers (including characters, figures, images, sound, animation and so on), the application of computer-aided multimedia technique can improve the effect of solfeggio in traditional teaching. Computers can be used to collect, analyze, edict and process multiple media. Multi-media technique means computers featuring interaction, integration and real time are used to process sound, characters and images and so on. During the solfeggio teaching, audio media technique is often used, plus the rapid development of stave software will make up for the sight reading, which make music "audiovisual synchronization", placing a good foundation for a wide application of multimedia technique in music. This article is focused on audio media related to the computer-aided multimedia technique. At the time of computer X86 in the 1990 s, we just predict the future of multimedia in theory, but today after about 20 years, especially with the wide spread of Internet, multimedia technique has been skillfully used in current teaching.

\section{FOUNDATION OF MULTIMEDIA APPLICATION}

During the solfeggio teaching, the sound technique in multimedia technique is mainly involved in MIDI and audio.

\section{A. MIDI}

MIDI is an abbreviation of Musical Instrument Digital Interface. In the 1980s, at the beginning of MIDI technique, the whole system consists of computer, sequencer software, MIDI keyboard or electronic synthesizer, sound source, sound console, playback device, digital voice recorder and so on. The whole system control center is computer, and peripheral units are connected through MIDI and audio wires, and the command information from instrument keyboard can be turned into digital information to save in computers, and send MIDI command to make the sound sources sound. Nowadays, the whole MIDI system include only a computer equipped with professional sound card and MIDI keyboard, such technical progress benefits from the progress of audio sampling technique. The sampling sound sources have replaced traditional electronic synthesizer and digital sound sources, and the sound console on sequencer software interface and digital audio integration of sound card port have replaced traditional sound console. Such progress has not only saved hardware costs but also simplified complex MIDI system and equipment size, the most important is the difficulties lowered for operation, making the MIDI system more practical and 
easy to operate. Computers and MIDI keyboards can form a system, and there are all kinds of software and sound cards for computers which can meet diverse teaching modes and goals.

\section{B. Audio Frequency}

Virtual instruments technique is the greatest invention in audio technique, which mainly relies on three techniques: ASIO drive mode and VSTi, DXi, all of the techniques have greatly improved the application of audio frequency in music.

VSTi is an abbreviation of Virtual Studio Technology Instruments, which is based on Steinberg, used through plug-in, able to use in most professional music software; DXi is an abbreviation of DirectX Instrument, a soft synthesizer technique developed by Cakewalk on the basis of DirectX, which can be used as plug-in or independently. VSTi and DXi are two techniques used on different platforms. Before appearance of the technique, Wavetable technique (the former software synthesizer) cannot be used in professional environment, and the software and hardware components in MIDI systems are very complicated, it is difficult to operate and teach as well.

\section{APPLICATION OF MUlTiMEDIA TECHNIQUE IN TRADITIONAL TRAINING}

Traditional solfeggio teaching is mainly focused on intonation and rhythm training:

\section{A. Intonation}

Piano tone is a basic content for each computer, and MIDI keyboard can complete replace piano keyboard, whose intonation is kept accurate absolutely, unnecessary for calibration. The octave adjustment up and down can make the keyboard keep about 61 keys, which greatly saves spaces in class, and each student can operate a keyboard. With regard to tuning systems, it is fit for learners with different professional orientation, such as just intonation of western strings instruments, Pythagorean tuning of folk musical instruments, as well as Equal Temperaments required by piano, accordion or other keyboard instruments, all of which can be solved through multimedia software. Software such as Audition and WaveLab can be used to reset pitch and different musical scales, and the intonation can be adjusted to cent, the powerful function make it easy for teachers to borrow the multimedia systems from teaching to acoustic research.

\section{B. Rhythm}

According to the initial MIDI protocol, the 10th audio track must be set to rhythm, which places a sound foundation for the rhythm application, later with the software development, the rhythm track is not limited in number as well as the appearance of folk percussion timbre libraries, all of these have made it more convenient for the rhythm application. Even professional rhythm software is developed such as FL STUDIO, which have created conditions for rhythm training. Modern music and JAZZ music both take on complex rhythms and multivoice compound rhythm, which can be used for training through software support.

\section{EXPANSION OF MULTIMEDIA IN TRADITIONAL TRAINING}

Timbre, harmony and music style training are uneasy to train in traditional solfeggio teaching, which at present has been solved through multimedia.

\section{A. Timbre}

Nowadays, during the course of teaching, any sound card which supports ASIO (Audio Stream Input Output standard, raised by Steinberg) can be used to offer high quality sound effect. In early years of multimedia, the sound delay (namely dull response by keyboard) was a serious flaw, which now has been completely solved by AISO. To support ASIO drive has also become a basic indicator for modern sound cards.

With the application of visual instruments such as VSTi and DXi and technical difficulties solved by ASIO drive, the reproduction of timbre has been a piece of cake for multimedia computers. Modern audio technology makes it easy for us to get any instruments through clicking. With regard to the timbre quality, the sound produced by computer is almost the same as that of actual musical instruments, which breaks through the initial simulation stage. If you want to reproduce some timbre, a computer is enough, in class teaching, the computer can be used to get any musical instrument and timbre for timbre training in solfeggio.

The progress of MIDI and audio technology has enabled us to break through the limitations in piano timbre in teaching, expanding the intonation training to all kinds of musical instruments, especially for western musical instruments, the quality of sampled timbre just seems like those from real musical instruments. With a computer, we can really make all kinds of sounds in class, for example, the complex sound source Hypersonic 2.0, 2G, contain all GM timbres and 9 drum sets, occupying a few hardware and memory, besides, it may produce authentic timbre, able to install on many computers as plug-in. Computers with higher configuration will have more professional timbres such as diverse versions of orchestral music plug-in East West Quantum Leap, Vienna orchestral music timbre library, hundreds of $\mathrm{G}$ timbres, all of which can reproduce the original sound of musical instruments.

\section{B. Harmony}

During the multi-voice training in solfeggio teaching, under the traditional teaching mode, generally students are arranged to act as different parts and achieve them through piano. However, the piano playing will restrict the contents teachers teach. Sequencer software are based multi-voice music, and the music instruments can be set to 128 channels or more, that's to say, 128 different parts of musical instruments at most can be loaded at the same time, which have greatly exceeded the number required for teaching, besides, it is easy to operate, for two or three timbres, it can be set in situ; if more complicated parts are needed, before class teaching, teachers can make preparations, setting in advance multiple parts, the music and timbres of several musical instruments, and integrating multi-voice training to the class teaching. Composing software such as Sibelius and Finale have visually represented stave interface, making students see pitch and 
rhythm. For example, in the software Sibelius, the interface of piano great stave can be used in situ for composing musical notes, from which students can directly feel the pitch; on the music score of string quartet, we can directly get the timbre combination of violin and cello in different ranges.

\section{Music Style}

With regard to music styles, the applications of computer software and hardware have brought greater help to teachers and students get music styles in different areas and times. For example, the JAZZ training in Earmaster include JAZZ chord composition, harmonic progression, rhythm simulation training and so on; In the writing mode of Sibelius, it is set with many templates, including classic, Jazz, modern, popular and film and even musical instruments templates of folk musical instruments from America and Africa; The applications of software have greatly improved teachers' professional quality, for example, generally teachers are not good at ranges and playing skills of different musical instruments, yet the software concerned can solve the problems easily in class, and students are helped directly feel all kinds of musical instruments from around the world, achieving all-sided knowledge about instrumental music and vocal music and improving students' music accomplishments.

\section{Changes in Teaching Management Mode}

Advanced teaching management can improve teaching quality and supplementary means in teaching. Much software while providing multiple forms for professional training has management tools making it easy for solfeggio teaching. Take the software Auralia as an example, it can be used to save or delete students' records, test results management, able to form a teaching network in LAN for exercises of several students; after students complete the training, it can immediately assess the results, and targeted training can be selected for students according to the knowledge level. The software Earmaster is designed with results statistics and ranking menu, which can create independent archives for class teaching and students' exercise results, saved in PDF; record the training time, contents and progress and assessment with words for a single student; results analysis and comparison of whole class students. Through the software, teachers can gasp what students have learned and make an orderly arrangement of students' learning progress and contents.

\section{EXPANSION OF COMPREHENSIVE EXERCISES}

The rapid developments of computer software and hardware have make teaching not be limited to some software or technique, and such teaching equipment need teachers to exert their enthusiasm, creativity and innovation.

Main characteristics of multimedia are interaction, integration and real time, during teaching, teachers shall create multiple interactive teaching environment: based on equal communications between teachers and students, opinions from different angles will impact with each other to stimulate students' enthusiasm and thirst for exploration, greatly improving the teaching efficiency. The advanced multimedia makes it possible to achieve the interaction between teaching and learning. Some software has audio technology integrated to MIDI, which can be fully used by teachers to change traditional teaching modes, achieving the interaction via software.

For example, Auralia, a typical interactive solfeggio teaching software, contains contents such as course teaching, quizzes, assessment, as well as teaching analysis and evaluation, and game modes are set in the software for answer first matches, knockout, cycling and other interest teaching, which can be used by teachers in class, besides, the excellent design will also attract students' self-learning and after-school counseling. Training contents include intervals, scales, rhythms, pitch melodies, chords, covering trainings of all musical abilities from classical music to modern one.

The software Earmaster is called master in ear training, which is focused on the integration training in listening such as intervals, chord, rhythm and melody. All stages are designed grade after grade, and sound-image training and Q\&A interaction have greatly improved students' interests in learning, reducing the difficulties in training in traditional solfeggio teaching. Different from traditional teaching where a teacher teaches a lot of students, such software makes it easy for students to exercise independently through computers, and real time training results will be shown to students to know about their progress and exercise contents, in addition, teachers may offer overall instruction and solve some problems through management system.

Composing software such as Sibelius and Finale are an embodiment of real time multimedia computers, the software can be used all-sided and fundamental music, main factors such as notes, rhythm, chord, mode and melody can be directly displayed on the screen, with correct listening effect and direct visual effect, the teaching contents are very clear. In case of any problems raised by students in class or specific problems, the software can be used for explanations.

\section{CONCLUSION}

The multimedia technique can keep the listening training data correct, flexible and all-sided in class; teaches can accurately adjust parameters of listening training data; the multimedia technique makes it possible to carry out multiple timber training, making exams quantitative, standard and impersonal, and the multimedia teaching is an efficient way to improve students' interests in training, renew traditional solfeggio teaching mode and improving training methods and contents.

\section{REFERENCES}

[1] The Computer Music Tutorial, [USA] Curtis Roads, People's Music Publishing House, May 2011

[2] The Being of Tone, An Introduction of Musical Acoustics, written by Han Baoqiang, People's Music Publishing House, Jan 2016.

[3] Solfeggio (Revision), written by Xu Jingxing, Sun Hong, Apr 2008, Higher Education Press. 